\section{SOI: $1.1 / \mathrm{TAS} \quad$ DOI: $10.15863 / \mathrm{TAS}$ \\ International Scientific Journal Theoretical \& Applied Science}

p-ISSN: 2308-4944 (print) e-ISSN: 2409-0085 (online)

Year: 2015 Issue: 05 Volume: 25

Published: $30.05 .2015 \quad$ http://T-Science.org
Anatoly Aleksandrovich Naumov Docent, Candidate of Technical Sciences, Academician of International Academy TAS, Center of Applied Mathematical Research, Novosibirsk, Russia a_a_naumov@mail.ru

Anastasia Anatolievna Naumova Marketing Director, MBA, Marie Forleo Business School, NYC,

Manchester, UK anastasia.naumova@yahoo.com

SECTION 31. Economic research, finance, innovation, risk management.

\title{
ABOUT PROBLEMS OF DOSUZHEVA-KIRILLOV MODELS OF INVESTMENT PROJECTS ANALYSIS AND THEIR PERMISSION WAYS
}

\begin{abstract}
The paper discusses approaches to effectiveness of investment projects estimating taking into account inflation. Considered classic efficiency indicators NPV, IRR, DPP, etc.. Identified the weaknesses of some schemes to evaluate the effectiveness of projects subject to the inflation factor. Identified the weaknesses of some schemes analysis of the accuracy of the payback period estimates. Investigated some problems of optimization models for synthesis of projects. Proposed approaches to the evaluation of the effectiveness of integrated projects. Identified issues the proposed methods use a systematic approach to the analysis of projects.

Key words: Investment projects, efficiency, inflation, efficiency criteria, NPV, IRR, DPP, optimization, integrated projects.

Language: Russian

Citation: Naumov AA, Naumova AA (2015) ABOUT PROBLEMS OF DOSUZHEVA-KIRILLOV MODELS OF INVESTMENT PROJECTS ANALYSIS AND THEIR PERMISSION WAYS. ISJ Theoretical \& Applied Science 05 (25): 110-118.

Soi: $\underline{\text { http://s-o-i.org/1.1/TAS*05(25)20 Doi: crossef http://dx.doi.org/10.15863/TAS.2015.05.25.20 }}$
\end{abstract}

\section{УДК 330.322.011:330.322.5}

\section{О ПРОБЛЕМАХ МОДЕЛЕЙ ДОСУЖЕВОЙ-КИРИЛЛОВА АНАЛИЗА ИНВЕСТИЦИОННЫХ ПРОЕКТОВ И ПУТЯХ ИХ РАЗРЕШЕНИЯ}

Аннотация: В работе рассмотрены подходы к оцениванию эффективности инвестиционных проектов с учетом инфляции. Рассмотрены классические показатели эффективности NPV, IRR, DPP u др.. Выявлены недостатки некоторых схем анализа эффективности проектов с учетом фактора инфляции. Выявлены недостатки некоторых схем оценивания срока окупаемости проектов. Исследованы проблемы некоторых оптимизационных моделей для синтеза проектов. Предложены подходы к оцениванию эффективности интегрированных проектов. Выявлены проблемь предлагаемых методик использования системного подхода к анализу проектов.

Ключевые слова: инвестиционные проекты, эффективность, инфляция, критерии эффективности, NPV, IRR, DPP, оптимизачия, интегрированные проекты.

\section{Введение}

Методы анализа и синтеза инвестиционных проектов (ИП) хорошо известны и находят широкое применение при решении многих реальных задач, связанных с выбором эффективных схем инвестирования (см. [1]-[8]).

Поводом для написания данной работы послужили модели, предлагаемые Е.Е. Досужевой и Ю.В. Кирилловым в их работах и в частности - модель, предложенная в работе [9] и предназначенная для оценивания эффективности инвестиционных проектов с учетом инфляционной составляющей. Казалось бы, этот вопрос достаточно хорошо изучен (см. [3], [7], [8]) и предложены рекомендации по практическому использованию схем анализа таких проектов. Однако желание предложить «новые» модели для решения этих задач часто приводят к тому, что эти «новые» модели отдаляют их от практического использования. Другими словами, модификации существующих моделей приводят к моделям, которыми 
пользоваться на практике не рекомендуется в силу того, что они содержат некорректности. Особенно остро это может проявиться в тех случаях, когда такие модели будут рекомендованы к практическому использованию, будут заложены в справочники, руководства, реализованы в виде пакетов программ для компьютеров.

Начнем изложение с проблем модели оценивания эффективности инвестиционных проектов с учетом инфляционных процессов.

Оценивание показателям процессов

Пусть инвестиционный проект (ИП) задан своими входным и выходным финансовыми потоками: $\quad F_{\text {in }}(t), \quad t=t_{0}, t_{1}, t_{2}, \ldots,\left(t_{m}=T\right), \quad-$ входной и $\mathrm{F}_{\text {out }}(\mathrm{t}), \mathrm{t}=\mathrm{t}_{0}, \mathrm{t}_{1}, \mathrm{t}_{2}, \ldots,\left(\mathrm{t}_{\mathrm{m}}=\mathrm{T}\right), \quad-$ выходной потоки соответственно. Пусть моменты времени $t_{0}, t_{1}, t_{2}, \ldots,\left(t_{m}=T\right)$ выбраны так, что интервалы между соседними отсчетами равны единице времени, например, без умаления общности ниже будем полагать $\mathrm{t}_{0}=0, \mathrm{t}_{1}=$ $1, \mathrm{t}_{2}=2, \ldots,\left(\mathrm{t}_{\mathrm{m}}=\mathrm{T}=m\right)$.

Положим, что эффективность ИП на момент времени $\mathrm{t} \quad\left(\mathrm{t} \in\left\{\mathrm{t}_{0}, \mathrm{t}_{1}, \mathrm{t}_{2}, \ldots,\left(\mathrm{t}_{\mathrm{m}}=\mathrm{T}\right)\right\}\right)$ оценивается показателем NPV (Net Present Value) следующим образом:

$$
\begin{aligned}
& N P V(t)=F_{\text {out }}^{\Sigma}(t)-F_{\text {in }}^{\Sigma}(t) ; \\
& F_{\text {out }}^{\Sigma}(t)=\sum_{\tau=t_{0}}^{\mathrm{t}} F_{\text {out }}(\tau) /(1+r)^{\tau-t_{0}} ; \\
& F_{\text {in }}^{\Sigma}(\mathrm{t})=\sum_{\tau=t_{0}}^{\mathrm{t}} \mathrm{F}_{\text {in }}(\tau) /(1+\mathrm{r})^{\tau-\mathrm{t}_{0}} .
\end{aligned}
$$

В (1) используется ставка дисконтирования финансовых потоков $r$, a $t_{0}$ - точка приведения потоков. При использовании (1) обычно полагают $\mathrm{t}=\mathrm{T}$.

В работе [9] для оценивания показателя эффективности проектов NPV в условиях инфляционных процессов $\mathrm{NPV}_{\text {inf }}$ предлагается использовать следующую схему (модель, назовем ее моделью Досужевой-Кириллова, МДК ${ }_{\mathrm{INF}}$ ):

$$
\begin{aligned}
& \mathrm{NPV}_{\text {inf }}(T)=F_{\text {out }, \text { inf }}^{\Sigma}(T)-F_{\text {in }, \text { inf }}^{\sum}(T) ; \\
& F_{\text {out }, \text { inf }}^{\Sigma}(T)=\sum_{\tau=t_{0}}^{T} F_{\text {out }}(\tau)(1+ \\
& \left.+r_{\text {inf }, \mathrm{F}_{\text {in }}, \mathrm{F}_{\text {out }}}\right)^{\tau-t_{0}} /(1+r)^{\tau-t_{0}} \\
& F_{\text {in }, \text { inf }}^{\Sigma}(T)=\sum_{\tau=t_{0}}^{T} F_{\text {in }}(\tau)(1+
\end{aligned}
$$$$
\left.r_{\text {inf }, \mathrm{F}_{\text {in }}, \mathrm{F}_{\text {out }}}\right)^{\tau-t_{0}} /(1+r)^{\tau-t_{0}} \text {. }
$$

Здесь $r_{\text {inf, } \mathrm{F}_{\mathrm{in}}, \mathrm{F}_{\mathrm{out}}}-$ темп инфляции (ставка, уровень инфляции) [3] общий для входного и выходного финансовых потоков проекта. Другими словами, если обозначить через $\mathrm{r}_{\text {inf }} \mathrm{F}_{\mathrm{in}}$, $\mathrm{r}_{\text {inf, }} \mathrm{F}_{\text {out }}$ темпы инфляции для $\mathrm{F}_{\text {in }}(\mathrm{t})$ и $\mathrm{F}_{\text {out }}(\mathrm{t})$ соответственно, то $r_{\text {inf, }} \mathrm{F}_{\mathrm{in}}, \mathrm{F}_{\mathrm{out}}-$ единый (общий) темп инфляции для обоих потоков, т.е. в [9] полагается, что выполняется равенство $\mathrm{r}_{\text {inf, }} \mathrm{F}_{\text {in }}=$ $\mathrm{r}_{\text {inf }, \mathrm{F}_{\text {out }}}=r_{\text {inf } f, \mathrm{~F}_{\text {in }}, \mathrm{F}_{\text {out }}}$.
Заметим, что в МДК INF [9] эффективность проектов оценивается для случая так называемых стандартных финансовых потоков. Для них характерно то, что на начальных этапах реализации ИП осуществляются только вложения средств, а затем получают доход без вложений в ИП финансовых средств. Очевидно, такие проекты встречаются на практике очень редко, т.к. получение доходов в проектах предполагает определенные расходы, связанные с их сопровождением.

Отметим основные недостатки модели МДК ${ }_{\text {INF }}$ [9].

Использование общего индекса инфляции $r_{\text {inf }}, \mathrm{F}_{\text {in }}, \mathrm{F}_{\text {out }}$ в модели (2) для входного и выходного финансовых потоков проекта $\left(\mathrm{F}_{\text {in }}(\mathrm{t})\right.$ и $\mathrm{F}_{\text {out }}(\mathrm{t}), \mathrm{t} \in$ $\left.\left\{\mathrm{t}_{0}, \mathrm{t}_{1}, \mathrm{t}_{2}, \ldots,\left(\mathrm{t}_{\mathrm{m}}=\mathrm{T}\right)\right\}\right) \quad$ не соответствует реальностям функционирования (реализации) проектов. Очевидно, что инфляция в разной степени влияет на элементы этих потоков.

Хорошо известно, что ставка дисконтирования $r$ зависит от инфляционных процессов. И для нее существует свой темп инфляции, обозначим его как $r_{i n f}$.

В общем случае темпы инфляции зависят от времени $t$ (являются динамическими), т.е. в расчетных формулах (и в модели МДК конечно же, тоже) они должны быть представлены в виде: $r_{\text {inf, } \mathrm{F}_{\text {in }}, \mathrm{F}_{\text {out }}}(t)$ и $r_{\text {inf }}(t)$.

В [9] сделана попытка исследовать показатель $N P V_{i n f}(T)$ на чувствительность и на эффективность (положительность его значений при изменении темпа $\left.r_{\text {inf }}, \mathrm{F}_{\text {in }}, \mathrm{F}_{\text {out }}\right)$. Но, сделано это некорректно, т.к. условия положительности $N P V_{\text {inf }}(T)$ представлены в [9] в виде неравенств $F_{\text {out,inf }}^{\Sigma}(T)>F_{\text {in,inf }}^{\Sigma}(T) \quad$ (которые получены очевидным образом из неравенства $N P V_{\text {inf }}(T)=$ $\left.F_{\text {out }, \text { inf }}^{\Sigma}(T)-F_{\text {in,inf }}^{\Sigma}(T)>0\right)$. Что касается исследования показателя $N P V_{\text {inf }}(T)$ на чувствительность, то это предполагает изучение изменений отношений вида $\Delta N P V_{\text {inf }}(T) / \Delta r_{\text {inf }}$ в зависимости от изменений значений приращений $\Delta r_{\text {inf }}$. Здесь $\Delta N P V_{\text {inf }}(T)$ - изменение показателя $N P V_{\text {inf }}(T), \quad$ обусловленное изменением $\Delta r_{i n f}$ (темпа инфляции $r_{i n f}$ ). В общем случае необходимо было исследовать на чувствительность показатель $\quad N P V_{\text {inf }}(T)$ относительно динамических ставок $r_{i n f}(t)$, $r_{\text {inf }, F_{\text {in }}}(t), \quad r_{\text {inf, } F_{\text {out }}}(t), \quad t=t_{0}, t_{1}, t_{2}, \ldots,\left(t_{m}=T\right)$. Эта задача достаточно сложная и с практической точки зрения представляет большой интерес. Ее решение позволит ранжировать параметры инфляции по степени их влияния на показатели эффективности проектов (для бизнес-процессов решение таких задач подробно исследовано, например, в [19], см. также [20]). 
Еще одно замечание. Исследование проектов на эффективность при наличии инфляции и при ее отсутствии следует проводить по единой схеме. Если необходимо исследовать проект в условиях отсутствия инфляции (а вдруг!), то следует положить: $r_{\text {inf }, \mathrm{F}_{\text {in }}, \mathrm{F}_{\text {out }}}(t)=0$, $r_{\text {inf }}(t)=0, \quad r_{\text {inf }, F_{\text {in }}}(t)=0, \quad r_{\text {inf }, F_{\text {out }}}(t)=0, \quad t=$ $t_{0}, t_{1}, t_{2}, \ldots,\left(t_{m}=T\right)$. При этом не нужно строить новые (дополнительные) схемы анализа проектов, как это предлагается делать в [9].

Устраним некорректности и преобразуем модель МДК INF (2) на общий случай учета инфляционных процессов (более общую и корректную модель обозначим как $\mathrm{MC}_{\mathrm{INF}}$ ). Для этого введем следующие определения и обозначения. Инфляция влияет на все элементы финансовых потоков ИП (на элементы входного и выходного финансовых потоков, на ставки дисконтирования и пр.). Это влияние различно в соответствии с местом и ролью этих элементов в ИП. Методы оценивания эффективности ИП в условиях инфляции хорошо известны и широко используются на практике (см., например, [3] [8]). Например, изменение цен на ресурсы (материалы, сырье, оборудование и пр.) ИП приводит к изменению элементов входного потока $\mathrm{F}_{\mathrm{in}}(\mathrm{t}), \mathrm{t}=\mathrm{t}_{0}, \mathrm{t}_{1}, \mathrm{t}_{2}, \ldots,\left(\mathrm{t}_{\mathrm{m}}=\mathrm{T}\right)$. В свою очередь, изменение спроса на производимую в рамках ИП продукцию - к изменению элементов выходного потока $\mathrm{F}_{\text {out }}(\mathrm{t}), \mathrm{t}=\mathrm{t}_{0}, \mathrm{t}_{1}, \mathrm{t}_{2}, \ldots,\left(\mathrm{t}_{\mathrm{m}}=\right.$ T). Кроме этого, очевидно, инфляционные процессы влияют на изменение ставок (например, ставки дисконтирования $r$ ).

Будем полагать, что темпы инфляции (ставки, уровни инфляции) [3] соответствующие элементам финансовых потоков и ставкам известны и обозначены как $\mathrm{r}_{\text {inf }}$ (для $\mathrm{r}$ ), $\mathrm{r}_{\text {inf, } \mathrm{F}_{\text {in }}}$ (для $\mathrm{F}_{\text {in }}(\mathrm{t})$ ), $\mathrm{r}_{\text {inf }, \mathrm{F}_{\text {out }}}$ (для $\mathrm{F}_{\text {out }}(\mathrm{t})$ ). В общем случае темпы инфляции изменяются со временем, т.е. имеют вид: $\mathrm{r}_{\text {inf }}(t), \mathrm{r}_{\text {inf, } \mathrm{F}_{\text {in }}}(t), \mathrm{r}_{\text {inf, } \mathrm{F}_{\text {out }}}(t), \mathrm{t}=$ $\mathrm{t}_{0}, \mathrm{t}_{1}, \mathrm{t}_{2}, \ldots,\left(\mathrm{t}_{\mathrm{m}}=\mathrm{T}\right)$. Базисные индексы инфляции для соответствующих элементов финансовых потоков и ставок ИП можно определить следующим образом [3]:

1) $\mathrm{I}_{\text {inf }, \mathrm{F}_{\mathrm{in}}}(\mathrm{t})=\prod_{\tau=\mathrm{t}_{0}}^{\mathrm{t}} \mathrm{I}_{\text {inf }, \mathrm{F}_{\mathrm{in}}, \mathrm{c}}(\tau)$,

здесь $I_{\text {inf }, \mathrm{F}_{\mathrm{in}}, c}(\tau), \quad \tau=\mathrm{t}_{0}, \mathrm{t}_{1}, \mathrm{t}_{2}, \ldots,\left(\mathrm{t}_{\mathrm{m}}=\mathrm{T}\right), \quad$ - цепные общие индексы инфляции («c» - от английского «circuit») для элементов входного потока $\mathrm{F}_{\text {in }}(\mathrm{t})$, $\mathrm{t}=\mathrm{t}_{0}, \mathrm{t}_{1}, \mathrm{t}_{2}, \ldots,\left(\mathrm{t}_{\mathrm{m}}=\mathrm{T}\right)$ (отражают изменение элементов входного потока, т.е. затрат, за счет изменения цен на оборудование, сырье, рабочую силу, ставок кредитования и т.д.);

2) $\mathrm{I}_{\text {inf }, \mathrm{F}_{\text {out }}}(\mathrm{t})=\prod_{\tau=\mathrm{t}_{0}}^{\mathrm{t}} \mathrm{I}_{\text {inf }, \mathrm{F}_{\text {out }}, \mathrm{c}}(\tau), \quad$ здесь $I_{\text {inf }, \mathrm{F}_{\text {out },}}(\tau), \quad \tau=\mathrm{t}_{0}, \mathrm{t}_{1}, \mathrm{t}_{2}, \ldots,\left(\mathrm{t}_{\mathrm{m}}=\mathrm{T}\right)$, - цепные общие индексы для элементов выходного потока $\mathrm{F}_{\text {out }}(\mathrm{t}), \quad \mathrm{t}=\mathrm{t}_{0}, \mathrm{t}_{1}, \mathrm{t}_{2}, \ldots,\left(\mathrm{t}_{\mathrm{m}}=\mathrm{T}\right) \quad$ (отражают изменение цен на производимую в рамках ИП продукцию, изменение спроса на продукцию и т.д.);

3) и т.д.

Очевидно, в терминах темпов (уровней, норм, ставок) инфляции $\left(\mathrm{r}_{\text {inf, in }}(t), \mathrm{r}_{\text {inf, out }}(t)\right.$, $\mathrm{r}_{\text {inf }}(t), \quad \mathrm{r}_{\text {inf, } \mathrm{F}_{\text {in }}}(t), \quad \mathrm{r}_{\text {inf, } \mathrm{F}_{\text {out }}}(t), \quad \mathrm{t}=$ $\mathrm{t}_{0}, \mathrm{t}_{1}, \mathrm{t}_{2}, \ldots,\left(\mathrm{t}_{\mathrm{m}}=\mathrm{T}\right)$, и т.д.) базисные индексы инфляции можно представить следующим образом [3]:

1) $\mathrm{I}_{\text {inf, } \mathrm{F}_{\text {in }}}(\mathrm{t})=\prod_{\tau=\mathrm{t}_{0}}^{\mathrm{t}}\left(1+\mathrm{r}_{\text {inf }, \mathrm{F}_{\mathrm{in}}}(\tau)\right)$, здесь $\mathrm{r}_{\text {inf }, \mathrm{F}_{\text {in }}}(\tau), \tau=\mathrm{t}_{0}, \mathrm{t}_{1}, \mathrm{t}_{2}, \ldots, \mathrm{t}$, - темпы инфляции для элементов входного финансового потока $\mathrm{F}_{\text {in }}(\mathrm{t}), \mathrm{t}=\mathrm{t}_{0}, \mathrm{t}_{1}, \mathrm{t}_{2}, \ldots,\left(\mathrm{t}_{\mathrm{m}}=\mathrm{T}\right)$;

2) $\mathrm{I}_{\text {inf }, \mathrm{F}_{\text {out }}}(\mathrm{t})=\prod_{\tau=\mathrm{t}_{0}}^{\mathrm{t}}\left(1+\mathrm{r}_{\text {inf }, \mathrm{F}_{\text {out }}}(\tau)\right)$,

здесь $\mathrm{r}_{\text {inf }, \mathrm{F}_{\text {out }}}(\tau), \quad \tau=\mathrm{t}_{0}, \mathrm{t}_{1}, \mathrm{t}_{2}, \ldots,\left(\mathrm{t}_{\mathrm{m}}=\mathrm{T}\right), \quad-$ темпы инфляции для элементов выходного финансового потока $\mathrm{F}_{\text {out }}(\mathrm{t}), \mathrm{t}=\mathrm{t}_{0}, \mathrm{t}_{1}, \mathrm{t}_{2}, \ldots,\left(\mathrm{t}_{\mathrm{m}}=\right.$ T);

3) и т.д.

Тогда выражения для показателя NPV c учетом инфляционной составляющей можно записать на основе вышеприведенных выражений (2), в которых необходимо использовать потоки и ставки с учетом темпов инфляции: $\mathrm{F}_{\text {in }}\left(\mathrm{t}, \mathrm{r}_{\text {inf }, \mathrm{F}_{\text {in }}}(t)\right), \quad \mathrm{F}_{\text {out }}\left(\mathrm{t}, \mathrm{r}_{\text {inf }, \mathrm{F}_{\text {out }}}(t)\right), \quad \mathrm{t}=$ $t_{0}, t_{1}, t_{2}, \ldots,\left(t_{m}=T\right)$ и т.д. Тогда выражения для показателя NPV (на момент времени $t \quad(t \in$ $\left.\left\{\mathrm{t}_{0}, \mathrm{t}_{1}, \mathrm{t}_{2}, \ldots,\left(\mathrm{t}_{\mathrm{m}}=\mathrm{T}\right)\right\}\right)$ ) примут вид (модель $\left.\mathrm{MC}_{\mathrm{INF}}\right)$ :

$$
\begin{aligned}
& \mathrm{NPV}_{\text {inf }}(\mathrm{t})=\mathrm{F}_{\text {out }, \text { inf }}^{\Sigma}(\mathrm{t})-\mathrm{F}_{\text {in }, \text { inf }}^{\sum}(\mathrm{t}) ; \\
& \mathrm{F}_{\text {out }, \text { inf }}^{\Sigma}(\mathrm{t})= \\
& \sum_{\tau=\mathrm{t}_{0}}^{\mathrm{t}} \frac{\mathrm{F}_{\text {out }}\left(\tau, \mathrm{r}_{\text {inf }, \mathrm{F}_{\text {out }}}(\tau)\right)}{\left(\prod_{\delta=\mathrm{t}_{0}}^{\tau-\mathrm{t}_{0}}(1+\mathrm{r}(\delta)) \cdot \Pi_{\gamma=\mathrm{t}_{0}}^{\tau-\mathrm{t}_{0}}\left(1+\mathrm{r}_{\text {inf }}(\gamma)\right)\right)} ; \\
& \mathrm{F}_{\text {in }, \text { inf }}^{\sum}(\mathrm{t})= \\
& \sum_{\tau=\mathrm{t}_{0}}^{\mathrm{t}} \frac{\mathrm{F}_{\text {in }}\left(\tau, \mathrm{r}_{\text {inf }, \mathrm{F}_{\text {in }}}(\tau)\right)}{\left(\prod_{\delta=\mathrm{t}_{0}}^{\tau-\mathrm{t}_{0}}(1+\mathrm{r}(\delta)) \cdot \prod_{\gamma=\mathrm{t}_{0}}^{\tau-\mathrm{t}_{0}}\left(1+\mathrm{r}_{\text {inf }}(\gamma)\right)\right)}
\end{aligned}
$$

Здесь полагается, что $\mathrm{r}_{\text {inf }}\left(\mathrm{t}_{0}\right)=\mathrm{r}_{\text {inf }}(0)=0$.

Заметим, что в (3) использован базисный индекс инфляции для ставки дисконтирования в виде $\prod_{\gamma=\mathrm{t}_{0}}^{\tau-\mathrm{t}_{0}}\left(1+\mathrm{r}_{\text {inf }}(\gamma)\right)$, который вычисляется через цепные индексы инфляции $\left(1+\mathrm{r}_{\text {inf }}(\gamma)\right)$, $\gamma \in\left\{\mathrm{t}_{0}, \mathrm{t}_{1}, \mathrm{t}_{2}, \ldots, \tau-\mathrm{t}_{0}\right\}$.

Если известно, что темпы инфляции постоянны и не зависят от времени, то выражения для вычисления $\mathrm{NPV}_{\text {inf }}(\mathrm{t}) \quad(\mathrm{t} \in$ $\left\{\mathrm{t}_{0}, \mathrm{t}_{1}, \mathrm{t}_{2}, \ldots,\left(\mathrm{t}_{\mathrm{m}}=\mathrm{T}\right)\right\}$ ) упрощаются (упрощенная модель $\mathrm{MC}_{\mathrm{INF}}$ :

$$
\begin{aligned}
& \mathrm{NPV}_{\text {inf }}(\mathrm{t})=\mathrm{F}_{\text {out,inf }}^{\Sigma}(\mathrm{t})-\mathrm{F}_{\text {in,inf }}^{\Sigma}(\mathrm{t}) \text {; } \\
& \mathrm{F}_{\text {out }, \text { inf }}^{\Sigma}(\mathrm{t})=
\end{aligned}
$$

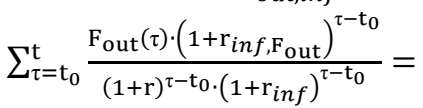




$$
\begin{aligned}
& \sum_{\tau=\mathrm{t}_{0}}^{\mathrm{t}} \mathrm{F}_{\text {out }}(\tau) \cdot\left(\frac{\left(1+\mathrm{r}_{\text {inf }}, \mathrm{F}_{\text {out }}\right)}{(1+\mathrm{r}) \cdot\left(1+\mathrm{r}_{\text {inf }}\right)}\right)^{\tau-\mathrm{t}_{0}} \text {; } \\
& \mathrm{F}_{\mathrm{in}, \text { inf }}^{\sum}(\mathrm{t})=\sum_{\tau=\mathrm{t}_{0}}^{\mathrm{t}} \frac{\mathrm{F}_{\mathrm{in}}(\tau) \cdot\left(1+\mathrm{r}_{\text {inf }, \mathrm{F}_{\text {in }}}\right)^{\tau-\mathrm{t}_{0}}}{(1+\mathrm{r})^{\tau-\mathrm{t}_{0}} \cdot\left(1+\mathrm{r}_{\text {inf }}\right)^{\tau-\mathrm{t}_{0}}}= \\
& \sum_{\tau=\mathrm{t}_{0}}^{\mathrm{t}} \mathrm{F}_{\mathrm{in}}(\tau) \cdot\left(\frac{\left(1+\mathrm{r}_{\text {inf }, \mathrm{F}_{\mathrm{in}}}\right)}{(1+\mathrm{r}) \cdot\left(1+\mathrm{r}_{\text {inf }}\right)}\right)^{\tau-\mathrm{t}_{0}} .
\end{aligned}
$$

Если предположить, что выполняются равенства $\mathrm{r}_{\text {inf, } \mathrm{F}_{\text {in }}}=\mathrm{r}_{\text {inf, } \mathrm{F}_{\text {out }}}=\mathrm{r}_{\text {inf }}$ (см. [3]), тогда будем иметь:

$$
\begin{gathered}
\mathrm{NPV}_{\text {inf }}(\mathrm{t})=\mathrm{F}_{\text {out,inf }}^{\Sigma}(\mathrm{t})-\mathrm{F}_{\text {in, inf }}^{\Sigma}(\mathrm{t}) ; \\
\left(\frac{1}{(1+\mathrm{r})}\right)^{\tau-\mathrm{t}_{0}}=\sum_{\tau=\mathrm{t}_{0}}^{\mathrm{t}} \cdot \frac{\mathrm{F}_{\text {out }}(\tau)}{(1+\mathrm{r})^{\tau-\mathrm{t}_{0}}} ;(5) \\
\mathrm{F}_{\mathrm{in}, \text { inf }}^{\Sigma}(\mathrm{t})=\sum_{\tau=\mathrm{t}_{0}}^{\mathrm{t}} \mathrm{F}_{\text {in }}(\tau) \cdot\left(\frac{1}{(1+\mathrm{r})}\right)^{\tau-\mathrm{t}_{0}}= \\
\sum_{\tau=\mathrm{t}_{0}}^{\mathrm{t}} \cdot \frac{\mathrm{F}_{\text {in }}(\tau)}{(1+\mathrm{r})^{\tau-\mathrm{t}_{0}}} \cdot \\
\mathrm{B} \mathrm{этом} \mathrm{случае} \mathrm{показатель} \mathrm{NPV}_{\text {inf }}(\mathrm{t}) \text { не }
\end{gathered}
$$
зависит от инфляции. Конечно, на практике подобные ограничения на темпы инфляции $\left(\mathrm{r}_{\text {inf }, \mathrm{F}_{\text {in }}}=\mathrm{r}_{\text {inf }, \mathrm{F}_{\text {out }}}=\mathrm{r}_{\text {inf }}\right)$ неприменимы и, к сожалению, упрощенными выражениями (5) воспользоваться на практике не представляется возможным.

Очевидным образом с учетом инфляции могут быть записаны и выражения для других критериев эффективности.

Для оценивания значения критерия IRR (внутренней норы доходности) c учетом инфляции (на основе формулы (4)) можно воспользоваться формулой (в рамках модели $\left.\mathrm{MC}_{\mathrm{INF}}\right)$ :

$$
\begin{gathered}
\operatorname{IRR}_{\text {inf }}(\mathrm{t})= \\
\left\{\begin{array}{l}
\mathrm{r} \mid \sum_{\tau=\mathrm{t}_{0}}^{\mathrm{t}} \mathrm{F}_{\text {in }}(\tau) \cdot\left(\frac{\left(1+\mathrm{r}_{\text {inf } \left., \mathrm{F}_{\text {in }}\right)}\right)}{(1+\mathrm{r}) \cdot\left(1+\mathrm{r}_{\text {inf }}\right)}\right)^{\tau-\mathrm{t}_{0}}= \\
=\sum_{\tau=\mathrm{t}_{0}}^{\mathrm{t}} \mathrm{F}_{\text {out }}(\tau) \cdot\left(\frac{\left(1+\mathrm{r}_{\text {inf }, \mathrm{F}_{\text {out }}}\right)}{(1+\mathrm{r}) \cdot\left(1+\mathrm{r}_{\text {inf }}\right)}\right)^{\tau-\mathrm{t}_{0}}
\end{array}\right\} .
\end{gathered}
$$

А для нахождения дисконтированного срока окупаемости ИП (DPP) можно воспользоваться выражением:

$$
\begin{gathered}
\operatorname{DPP}_{\text {inf }}(\mathrm{t})= \\
\left\{\begin{array}{c}
\min \mathrm{t}^{*} \in\left\{\mathrm{t}_{0}, \mathrm{t}_{1}, \mathrm{t}_{2}, \ldots, \mathrm{t}_{\mathrm{m}}=\mathrm{T}\right\} \\
\mathrm{NPV}_{\text {inf }}(\mathrm{t}) \geq 0, \\
\forall_{\mathrm{t}} \mathrm{t} \in\left\{\mathrm{t}^{*}, \mathrm{t}^{*}+1, \mathrm{t}^{*}+2, \ldots, \mathrm{t}_{\mathrm{m}}=\mathrm{T}\right\}
\end{array}\right\} .
\end{gathered}
$$

\section{Анализ точности оценки срока окупаемости проектов \\ В последнее время для оценивания} дисконтированного срока окупаемости DPP были предложены новые схемы расчета (см. подробнее в [10], [11]), причем авторами этих схем утверждается, что они позволяют оценить показатель DPP более точно, чем классические подходы, которые основаны на решении задачи:

$$
D P P=\left\{\begin{array}{c}
\min t^{*} \in\left\{t_{0}, t_{1}, t_{2}, \ldots, t_{m}\right\} \\
\operatorname{NPV}(t) \geq 0, \forall_{t} t \in\left[t^{*}, t_{m}\right]
\end{array}\right\} .
$$

Здесь $\mathrm{NPV}(\mathrm{t})$ имеет вид (1).

Точность классических методов нахождения DPP равна длине того интервала, правой границей которого служит значение этой оценки. Так, например, если $D P P=t^{*}=t_{k} \in$ $\left\{\mathrm{t}_{0}, \mathrm{t}_{1}, \mathrm{t}_{2}, \ldots, \mathrm{t}_{\mathrm{m}}\right\}$ (обозначение $\mathrm{t}^{*}$ соответствует решению задач (8)), то точность будет равна длине интервала $\left[\mathrm{t}_{\mathrm{k}-1}, \mathrm{t}_{\mathrm{k}}\right]$. Можно ли оценить срок окупаемости более точно? Ответ: к сожалению, нет! Это объясняется тем, что поведение потоков известно лишь в моменты времени $\left\{\mathrm{t}_{0}, \mathrm{t}_{1}, \mathrm{t}_{2}, \ldots, \mathrm{t}_{\mathrm{m}}\right\}$. Поведение потоков внутри интервала $\left[\mathrm{t}_{\mathrm{k}-1}, \mathrm{t}_{\mathrm{k}}\right]$ может быть самым различным и оно нам не известно. Поэтому, можно сказать, что точное значение срока окупаемости принадлежит этому интервалу и более точную оценку его, к сожалению, дать не представляется возможным. Можно ли повысить точность оценки критерия DPP? Да, для этого можно, например, уменьшить длины интервалов времени между точками множества $\left\{\mathrm{t}_{0}, \mathrm{t}_{1}, \mathrm{t}_{2}, \ldots, \mathrm{t}_{\mathrm{m}}\right\}$, при этом в новых точках разбиения мы должны знать значения финансовых потоков $\mathrm{F}_{\text {in }}(\mathrm{t})$ и $\mathrm{F}_{\text {out }}(\mathrm{t})$.

В модели Досужевой-Кириллова (назовем ее МДК прием, когда для повышения точности оценки показателя DPP дискретный поток доходов $\mathrm{F}_{\text {out }}(\mathrm{t}), \mathrm{t}=\mathrm{t}_{0}, \mathrm{t}_{1}, \mathrm{t}_{2}, \ldots, \mathrm{t}_{\mathrm{m}}$, заменяется на непрерывный поток и для случая стандартных потоков получается формула для срока окупаемости:

$$
\mathrm{DPP}=
$$

$-\ln \left\{1-\frac{\mathrm{s}\left(\mathrm{I}_{\mathrm{m}}\right)}{\sum_{\mathrm{k}=1}^{\mathrm{n}_{\mathrm{D}}} \frac{\mathrm{D}_{\mathrm{k}}}{(1+\mathrm{i})^{\mathrm{k}}}} \cdot\left(1-(1+\mathrm{i})^{-\mathrm{n}_{\mathrm{D}}}\right)\right\} / \ln (1+\mathrm{i})$.

Здесь $\mathrm{S}\left(\mathrm{I}_{\mathrm{m}}\right)$ - наращенная сумма инвестиций ИП ко времени окончания инвестиционного этапа $\left(\mathrm{t}=\mathrm{n}_{\mathrm{I}}\right) ; \mathrm{D}_{\mathrm{k}}-$ размеры доходов ИП по годам $\left(\mathrm{k}=1,2, \ldots, \mathrm{n}_{\mathrm{D}}\right) ; \mathrm{i} \quad-$ ставка дисконтирования (наращивания) потоков проекта.

B терминах данной работы $\mathrm{S}\left(\mathrm{I}_{\mathrm{m}}\right)=$ $\sum_{\mathrm{t}=\mathrm{t}_{0}}^{\mathrm{t}=\mathrm{t}_{\mathrm{I}}} \mathrm{F}_{\mathrm{in}}(\mathrm{t}) \cdot(1+\mathrm{r})^{\mathrm{t}_{\mathrm{n}_{\mathrm{I}}}-\mathrm{t}}, \quad \mathrm{r}=\mathrm{i} \quad-\quad$ ставка наращивания (дисконтирования) потоков, $\mathrm{F}_{\text {in }}=$ $\left(F_{\text {in }}\left(t_{0}\right), F_{\text {in }}\left(t_{1}\right), \ldots, F_{\text {in }}\left(t_{n_{I}}\right), 0,0, \ldots, 0\right)-$ вектор входного потока длиной в $\mathrm{m}+1=\mathrm{n}_{\mathrm{I}}+\mathrm{n}_{\mathrm{D}}+1$ элемент, причем, первые $\mathrm{n}_{\mathrm{I}}+1$ элемент его относятся к ненулевым вложениям в проект, а остальные $-\mathrm{n}_{\mathrm{D}}$ элементов - к нулевым; $\mathrm{F}_{\text {out }}=$ $\left(0,0, \ldots, 0, \mathrm{~F}_{\text {out }}\left(\mathrm{t}_{\mathrm{n}_{\mathrm{I}}+1}\right)=\mathrm{D}_{1}, \mathrm{~F}_{\text {out }}\left(\mathrm{t}_{\mathrm{n}_{\mathrm{I}}+2}\right)=\right.$ $\left.\mathrm{D}_{2}, \ldots, \mathrm{F}_{\text {out }}\left(\mathrm{t}_{\mathrm{n}_{\mathrm{I}}+\mathrm{n}_{\mathrm{D}}}\right)=\mathrm{D}_{\mathrm{n}_{\mathrm{D}}}\right)-$ вектор выходного потока проекта.

Для аналитиков ИП было бы очень удобно иметь расчетные формулы вида (9). Ведь по ним можно сколь угодно точно находить оценку для 
DPP. Но, воспользоваться подобными формулами на практике нельзя. Дело в том, что формула (9) в рамках модели МДК переходе от дискретного потока $\left(\mathrm{F}_{\text {out }}\right)$ к непрерывному потоку (обозначим $\mathrm{F}_{\text {out }}^{\infty}$ ) при этом для них выполняется равенство $\operatorname{NPV}\left(\mathrm{t}_{\mathrm{n}_{\mathrm{I}}}, \mathrm{F}_{\text {out }}\right)=$ $\operatorname{NPV}\left(\mathrm{t}_{\mathrm{n}_{\mathrm{I}}}, \mathrm{F}_{\text {out }}^{\infty}\right)$. Здесь $\operatorname{NPV}\left(\mathrm{t}_{\mathrm{n}_{\mathrm{I}}}, \mathrm{F}_{\text {out }}\right)$ приведенный в точку $\mathrm{t}=\mathrm{t}_{\mathrm{n}_{\mathrm{I}}}$ поток $\mathrm{F}_{\text {out }}$. Аналогично и обозначение $\operatorname{NPV}\left(\mathrm{t}_{\mathrm{n}_{\mathrm{I}}}, \mathrm{F}_{\text {out }}^{\infty}\right)$, но для потока $\mathrm{F}_{\text {out }}^{\infty}$. Можно сказать, что потоки $\mathrm{F}_{\text {out }}$ и $\mathrm{F}_{\text {out }}^{\infty}$ эквивалентны между собой относительно $\operatorname{NPV}\left(\mathrm{t}_{\mathrm{n}_{\mathrm{I}}}, \mathrm{F}_{\text {out }}\right)$. Кстати, таких выходных потоков, эквивалентных исходному, существует бесконечно много. Но, только один из них будет соответствовать реальному выходному потоку данного ИП ( $\left.\mathrm{F}_{\text {out }}\right)$, а остальные отношения к проекту иметь не будут. И, таким образом, исходный проект при таком переходе будет существенно изменен. Переход от дискретного потока доходов к непрерывному приводит к совершенно другому ИП, а значит, и показатель DPP (в рамках модели МДК этого нового ИП.

Задача оптимизации инвестиционных проектов и ее аналитическое решение

Рассмотрим еще одну модель ДосужевойКириллова, представляющую собой задачу оптимизации инвестиционных проектов на основе показателей NPV, DPP, DPI (см. подробнее описание модели и процедуру ее упрощения в [12], [13]). Обозначим эту модель как МДКорт.

Суть оптимизационной задачи в соответствии с МДКорт состоит в следующем. Пусть известны потоки ИП: $\mathrm{x}_{1}, \mathrm{x}_{2}, \mathrm{x}_{3}, \ldots, \mathrm{x}_{\mathrm{n}_{1}}-$ входной поток (инвестиций) проекта; $\mathrm{n}_{1}-$ длительность этапа инвестирования в проект; $\mathrm{y}_{\mathrm{n}_{1}+1}, \mathrm{y}_{\mathrm{n}_{1}+2}, \ldots, \mathrm{y}_{\mathrm{n}_{1}+\mathrm{n}_{2}}$ - элементы выходного потока (доходов) проекта; $\mathrm{n}_{2}-$ длительность этапа получения доходов от проекта; i - ставка дисконтирования или наращивания потоков проекта.

Тогда в соответствии с МДКОРТ для ИП можно оценить показатели NPV, DPP, DPI (см. [13]):

1) чистый приведенный доход -

$\operatorname{NPV}\left(\mathrm{x}_{1}, \mathrm{x}_{2}, \mathrm{x}_{3}, \ldots, \mathrm{x}_{\mathrm{n}_{1}}, \mathrm{y}_{\mathrm{n}_{1}+1}, \mathrm{y}_{\mathrm{n}_{1}+2}, \ldots, \mathrm{y}_{\mathrm{n}_{1}+\mathrm{n}_{2}}, \mathrm{i}\right)=$ $\sum_{\mathrm{k}=\mathrm{n}_{1}+1}^{\mathrm{n}_{1}+\mathrm{n}_{2}} \frac{\mathrm{y}_{\mathrm{k}}}{(1+\mathrm{i})^{\mathrm{k}}}-\sum_{\mathrm{j}=1}^{\mathrm{n}_{1}} \frac{\mathrm{x}_{\mathrm{j}}}{(1+\mathrm{i})^{\mathrm{j}}}$

2) дисконтированный срок окупаемости $\operatorname{DPP}\left(\mathrm{x}_{1}, \mathrm{x}_{2}, \mathrm{x}_{3}, \ldots, \mathrm{x}_{\mathrm{n}_{1}}, \mathrm{y}_{\mathrm{n}_{1}+1}, \mathrm{y}_{\mathrm{n}_{1}+2}, \ldots, \mathrm{y}_{\mathrm{n}_{1}+\mathrm{n}_{2}}, \mathrm{i}\right)=$ $-\ln \left\{1-\frac{\mathrm{s}\left(\mathrm{x}_{\mathrm{j}}\right)}{\mathrm{P}\left(\mathrm{y}_{\mathrm{k}}\right)} \cdot\left(1-(1+\mathrm{i})^{-\mathrm{n}_{2}}\right)\right\} / \ln (1+\mathrm{i})$, где $\mathrm{S}\left(\mathrm{x}_{\mathrm{j}}\right)$ - сумма инвестиций, приведенная к моменту времени $\mathrm{t}=\mathrm{n}_{1}$,

$$
\mathrm{S}\left(\mathrm{x}_{\mathrm{j}}\right)=\mathrm{S}\left(\mathrm{x}_{1}, \mathrm{x}_{2}, \mathrm{x}_{3}, \ldots, \mathrm{x}_{\mathrm{n}_{1}}\right)=\sum_{\mathrm{j}=1}^{\mathrm{n}_{1}} \mathrm{x}_{\mathrm{j}}
$$

$(1+\mathrm{i})^{\mathrm{n}_{1}-\mathrm{j}}, \quad$ а $\mathrm{P}\left(\mathrm{y}_{\mathrm{k}}\right)$ - дисконтированная стоимость доходов, приведенная к моменту $\mathrm{t}=\mathrm{n}_{1}, \quad \mathrm{P}\left(\mathrm{y}_{\mathrm{k}}\right)=\mathrm{P}\left(\mathrm{y}_{\mathrm{n}_{1}+1}, \mathrm{y}_{\mathrm{n}_{1}+2}, \ldots, \mathrm{y}_{\mathrm{n}_{1}+\mathrm{n}_{2}}\right)=$ $\sum_{\mathrm{k}=\mathrm{n}_{1}+1}^{\mathrm{n}_{1}+\mathrm{n}_{2}} \frac{\mathrm{y}_{\mathrm{k}}}{(1+\mathrm{i})^{\mathrm{k}-\mathrm{n}_{1}}}$;

3) дисконтированный индекс доходности (рентабельность ИП) -

$\operatorname{DPI}\left(\mathrm{x}_{1}, \mathrm{x}_{2}, \mathrm{x}_{3}, \ldots, \mathrm{x}_{\mathrm{n}_{1}}, \mathrm{y}_{\mathrm{n}_{1}+1}, \mathrm{y}_{\mathrm{n}_{1}+2}, \ldots, \mathrm{y}_{\mathrm{n}_{1}+\mathrm{n}_{2}}, \mathrm{i}\right)=$ $\sum_{\mathrm{k}=\mathrm{n}_{1}+1}^{\mathrm{n}_{1}+\mathrm{n}_{2}} \frac{\mathrm{y}_{\mathrm{k}}}{(1+\mathrm{i})^{\mathrm{k}}} / \sum_{\mathrm{j}=1}^{\mathrm{n}_{1}} \frac{\mathrm{x}_{\mathrm{j}}}{(1+\mathrm{i})^{\mathrm{j}}}$.

Тогда оптимизация ИП может быть сведена к решению задачи:

$\operatorname{NPV}\left(\mathrm{x}_{1}, \mathrm{x}_{2}, \mathrm{x}_{3}, \ldots, \mathrm{x}_{\mathrm{n}_{1}}, \mathrm{y}_{\mathrm{n}_{1}+1}, \mathrm{y}_{\mathrm{n}_{1}+2}, \ldots, \mathrm{y}_{\mathrm{n}_{1}+\mathrm{n}_{2}}, \mathrm{i}\right) \rightarrow$ $\rightarrow \max$

$$
\begin{aligned}
& \operatorname{DPP}\left(\mathrm{x}_{1}, \mathrm{x}_{2}, \mathrm{x}_{3}, \ldots, \mathrm{x}_{\mathrm{n}_{1}}, \mathrm{y}_{\mathrm{n}_{1}+1}, \mathrm{y}_{\mathrm{n}_{1}+2}, \ldots, \mathrm{y}_{\mathrm{n}_{1}+\mathrm{n}_{2}}, \mathrm{i}\right) \\
& \rightarrow \min
\end{aligned}
$$

при ограничениях:

$$
\left\{\begin{array}{c}
1 \leq \sum_{\mathrm{k}=\mathrm{n}_{1}+1}^{\mathrm{n}_{1}+\mathrm{n}_{2}} \frac{\mathrm{y}_{\mathrm{k}}}{(1+\mathrm{i})^{\mathrm{k}}} / \sum_{\mathrm{j}=1}^{\mathrm{n}_{1}} \frac{\mathrm{x}_{\mathrm{j}}}{(1+\mathrm{i})^{\mathrm{j}}} \leq \mathrm{DPI} I_{\max }, \\
\mathrm{x}_{\min } \leq \mathrm{x}_{\mathrm{j}} \leq \mathrm{x}_{\max }, \\
\mathrm{y}_{\min } \leq \mathrm{y}_{\mathrm{k}} \leq \mathrm{y}_{\max }, \\
\mathrm{i}_{\min } \leq \mathrm{i} \leq \mathrm{IRR}_{0}, \\
\mathrm{x}_{\mathrm{j}}, \mathrm{y}_{\mathrm{k}}, \mathrm{i} \geq 0 .
\end{array}\right.
$$

Здесь $\mathrm{IRR}_{0}$ - верхняя граница для ставки $\mathrm{i}$.

Отметим некоторые особенности задачи (10)-(11) (модели МДКорт).

Хорошо известно, что показатели NPV и DPI являются зависимыми. Отсюда следует, что можно было оставить один из показателей, например NPV, а другой из модели МДКорт исключить.

Решение задачи (10)-(11) является тривиальным и оно имеет вид: $\mathrm{x}_{\mathrm{j}}=\mathrm{x}_{\mathrm{j}, \mathrm{min}}, \mathrm{j}=$ $1,2, \ldots, \mathrm{n}_{1}, \mathrm{y}_{\mathrm{k}}=\mathrm{y}_{\mathrm{k}, \max }, \mathrm{k}=\mathrm{n}_{1}+1, \mathrm{n}_{1}+2, \ldots, \mathrm{n}_{1}+$ $\mathrm{n}_{2}, \mathrm{i}=\mathrm{i}_{\min }$.

Задача (10)-(11) содержит выражение для показателя DPP, которое, фактически, не позволяет найти срок окупаемости данного проекта (см. замечания к модели МДК

Задача (10)-(11) поставлена некорректно, т.к. в общем случае выходной поток ИП (переменные $\left.\mathrm{y}_{\mathrm{n}_{1}+1}, \mathrm{y}_{\mathrm{n}_{1}+2}, \ldots, \mathrm{y}_{\mathrm{n}_{1}+\mathrm{n}_{2}}\right)$ зависит от входного потока (переменные $\mathrm{x}_{1}, \mathrm{x}_{2}, \mathrm{x}_{3}, \ldots, \mathrm{x}_{\mathrm{n}_{1}}$ ), а это означает, что в ограничения задачи (10)-(11) необходимо дополнительно ввести ограничения, связывающие переменные $\mathrm{x}_{1}, \mathrm{x}_{2}, \mathrm{x}_{3}, \ldots, \mathrm{x}_{\mathrm{n}_{1}}$ и $\mathrm{y}_{\mathrm{n}_{1}+1}, \mathrm{y}_{\mathrm{n}_{1}+2}, \ldots, \mathrm{y}_{\mathrm{n}_{1}+\mathrm{n}_{2}}$.

В силу того, что решение этой задачи находится тривиально (см. замечание выше), то остается открытым вопрос: за счет чего будут снижены затраты (издержки) ИП до оптимальных значений $\mathrm{x}_{\mathrm{j}}=\mathrm{x}_{\mathrm{j}, \mathrm{min}}, \mathrm{j}=1,2, \ldots, \mathrm{n}_{1}$ и увеличена его доходная часть до значений $\mathrm{y}_{\mathrm{k}}=\mathrm{y}_{\mathrm{k}, \max }, \mathrm{k}=\mathrm{n}_{1}+$ $1, \mathrm{n}_{1}+2, \ldots, \mathrm{n}_{1}+\mathrm{n}_{2}$ ? На практике обычно сначала решают вопрос о снижении издержек и увеличении доходов, а уже потом пересчитывают 
в соответствии с этими изменениями показатели. Как правило, снижение затрат $\left(\mathrm{x}_{\mathrm{j}}\right)$ связано с выбором новых источников финансирования, новых схем расчета по кредитам и т.д., а потому задача оптимизации становится дискретной и алгоритм ее решения сводится к перебору вариантов инвестиционных схем. Аналогично обстоит дело и с проблемой увеличения доходов $\left(\mathrm{y}_{\mathrm{k}}\right)$. Это можно осуществить, например, за счет повышения цен на производимую продукцию.

Еще одно замечание к модели МДК Ограничение сверху на значение рентабельности проекта DPI представляется излишним; кроме этого, его можно заменить на эквивалентное этому ограничению ограничение NPV $\geq 0$.

Исходя из экономического смысла, выполняются неравенства $\mathrm{x}_{\mathrm{j}, \min } \geq 0$ для всех $\mathrm{j}=$ $1,2, \ldots, \mathrm{n}_{1}$, и $\mathrm{y}_{\mathrm{k}, \min } \geq 0$ для всех $\mathrm{k}=\mathrm{n}_{1}+1, \mathrm{n}_{1}+$ $2, \ldots, \mathrm{n}_{1}+\mathrm{n}_{2}$, поэтому ограничения вида $\mathrm{x}_{\mathrm{j}} \geq$ $0, \mathrm{j}=1,2, \ldots, \mathrm{n}_{1}, \quad$ и $\quad \mathrm{y}_{\mathrm{k}} \geq 0, \mathrm{k}=\mathrm{n}_{1}+1, \mathrm{n}_{1}+$ $2, \ldots, \mathrm{n}_{1}+\mathrm{n}_{2}$, являются лишними. Аналогично обстоит дело и с ограничением для ставки дисконтирования і .

В соответствии с этими замечаниями, вид задачи (10)-(11) может быть упрощен:

$\operatorname{NPV}\left(\mathrm{x}_{1}, \mathrm{x}_{2}, \mathrm{x}_{3}, \ldots, \mathrm{x}_{\mathrm{n}_{1}}, \mathrm{y}_{\mathrm{n}_{1}+1}, \mathrm{y}_{\mathrm{n}_{1}+2}, \ldots, \mathrm{y}_{\mathrm{n}_{1}+\mathrm{n}_{2}}, \mathrm{i}\right) \rightarrow$

$\rightarrow \max$

при ограничениях:

$\left\{\begin{array}{c}\operatorname{NPV}\left(\mathrm{x}_{1}, \mathrm{x}_{2}, \mathrm{x}_{3}, \ldots, \mathrm{x}_{\mathrm{n}_{1}}, \mathrm{y}_{\mathrm{n}_{1}+1}, \mathrm{y}_{\mathrm{n}_{1}+2}, \ldots, \mathrm{y}_{\mathrm{n}_{1}+\mathrm{n}_{2}}, \mathrm{i}\right) \geq 0, \\ \mathrm{x}_{\mathrm{j}, \mathrm{min}} \leq \mathrm{x}_{\mathrm{j}} \leq \mathrm{x}_{\mathrm{j}, \max }, \mathrm{j}=1,2, \ldots, \mathrm{n}_{1}, \\ \mathrm{y}_{\mathrm{k}, \min } \leq \mathrm{y}_{\mathrm{k}} \leq \mathrm{y}_{\mathrm{k}, \text { max }}, \mathrm{k}=\mathrm{n}_{1}+1, \mathrm{n}_{1}+2, \ldots, \mathrm{n}_{1}+\mathrm{n}_{2}, \\ \mathrm{i}_{\min } \leq \mathrm{i} .\end{array}\right.$

Решение упрощенной задачи совпадает с решением исходной задачи: $\mathrm{x}_{\mathrm{j}}=\mathrm{x}_{\mathrm{j}, \mathrm{min}}, \mathrm{j}=$ $1,2, \ldots, \mathrm{n}_{1}, \mathrm{y}_{\mathrm{k}}=\mathrm{y}_{\mathrm{k}, \mathrm{max}}, \mathrm{k}=\mathrm{n}_{1}+1, \mathrm{n}_{1}+2, \ldots, \mathrm{n}_{1}+$ $\mathrm{n}_{2}, \mathrm{i}=\mathrm{i}_{\min }$.

\section{К проблемам модели управления совместными проектами \\ В модели Досужевой-Кириллова,} касающейся совместного управления ИП (модель МДК ${ }_{\mathrm{INT}}$ ) (см. подробнее описание модели и ее анализ в [14], [15]), представлен один из подходов к анализу эффективности проектов, реализуемых совместно. Такие проекты предлагается представить в виде структуры из пяти последовательных проектов: академических НИИ, прикладных НИИ, ВУЗов, малых инновационных предприятий и крупных промышленных организаций.

Для оценивания эффективности интеграционного проекта (включающего пять этапов), в рамках модели МДК воспользоваться формулой для NPV:

$\operatorname{NPV}\left(\mathrm{x}_{1}, \mathrm{x}_{2}, \mathrm{x}_{3}, \mathrm{x}_{4}, \mathrm{x}_{5}, \mathrm{y}_{5}\right)=\sum_{\mathrm{i}=1}^{5}\left(\mathrm{P}_{\mathrm{i}}^{+}(\mathrm{t}=0)-\right.$

$\left.-\mathrm{P}_{\mathrm{i}}^{-}(\mathrm{t}=0)\right)=\sum_{\mathrm{i}=1}^{5} \mathrm{NPV}_{\mathrm{i}}$.
Здесь $\mathrm{y}_{5}$ - доход пятого этапа. Однако задача оптимизации проекта с критерием (12) (на максимум) может быть представлена в виде пяти независимых оптимизационных подзадач (для каждого этапа в отдельности с критериями $\mathrm{NPV}_{\mathrm{i}}, \mathrm{i}=1,2, \ldots, 5$, и со своими наборами переменных). Таким образом, фактически модель МДК INT $_{\text {н }}$ описывает интеграционный (совместный) ИП, а проект, разбитый на последовательность независимых этапов. Можно заключить, что модель МДК INT соответствует пяти независимым проектам для каждого из пяти этапов. Но, тогда возникает ряд замечаний по поводу модели МДК INT:

1) если проекты независимы, то нет необходимости приводить все потоки всех проектов к одной временной точке $\mathrm{t}=0$, как это сделано для $\mathrm{NPV}_{\mathrm{i}}, \mathrm{i}=1,2, \ldots, 5$;

2) нет необходимости строить общую модель, т.к. каждая из составляющих ее подмоделей имеет на каждом из этапов достаточно простой вид;

3) для такой последовательности независимых проектов возможна ситуация, когда какой-либо из них может оказаться в более выгодных условиях и наоборот (в модели МДК отсутствуют связывающие подмодели уравнения или уравнения-связки).

Как следовало бы решать задачу оценивания эффективности интеграции в общем случае? Схема может быть, например, такой (см., например, [16], [17]). На первом этапе решения задачи интеграции выстраивается из отдельных ИП структура интеграционного проекта. Такая структура может иметь достаточно сложный вид c учетом возможных наложений реализаций частных проектов во времени, обмена между ними ресурсами и пр. Затем для полученной структуры интеграционного проекта оцениваются доход и доходность (рекомендуем для этого использовать критерии NFV, IRR с использованием идеи компаундирования потоков и метода детализации финансовых потоков [16]). Полученные значения этих показателей должны устроить всех потенциальных участников интеграции. После этого с использованием доходности интеграционного процесса в целом IRR находятся доходы частных проектов и их доходности. Если каждого из участников интеграции значения доходов и доходностей их проектов устраивают, то принимается решение о детализации и уточнении некоторых характеристик интеграционного процесса и о заключении соответствующих документов (договоров, регламентов и пр.). При желании можно оценить и риски интеграционного процесса в целом и частных проектов в отдельности. Известно, что с усложнением структуры проектов (а интеграция как раз и 
предполагает такое усложнение) риски увеличиваются. Объясняется это очень просто: в структуре интеграционного проекта отдельные элементы оказывают влияние друг на друга (через недопоставки объемов ресурсов, сбои в графиках выполнения

работ, недофинансирование и пр.).

\section{Проблемы одной методики использования системного подхода к анализу инвестиционных проектов}

В работе [21] рассмотрены вопросы использования системного подхода к задачам анализа ИП на эффективность и оптимизации их параметров. Тема работы достаточно актуальна, т.к. в настоящее время не существует достаточно ясного представления о выборе критериев эффективности ИП и, в связи с этим, методов их оптимизации. Конечно, использование такого мощного инструментария, каким является системный анализ, к задачам анализа и синтеза ИП - должно было улучшить качество принимаемых по проекту решений, повысить эффективность проектов и т.д. Однако предлагаемые в этой работе методы содержат множество неточностей и ошибок. Перечислим некоторые из них.

1) На рис. 1 оператор сравнения (почему-то обозначен знаком "+") имеет два выхода. Вопрос, в каких случаях осуществляется переход по каждому из них?

2) О каком "Синтезе" идет речь на рис. 1? Почему в результате получается "Модель системы"?

3) Что имелось в виду, когда автор утверждает: "Сложность изучаемой системы, в данном случае сложность системы оценки эффективности инвестиционной деятельности как средства для реализации инвестиционных проектов, приведет к тому, что в качестве критериев задачи оптимизации необходимо будет использовать несколько экономических показателей наиболее приоритетных с точки зрения целей исследования" (стр. 4)?

4) Думается, что утверждение "...Конечно, использование сложного математического аппарата для анализа модели в форме задачи оптимизации создает дополнительные трудности в работе экономиста-исследователя и в этом, на наш взгляд, как раз и заключена причина того, что такие экономико-математические модели нечасто используют на практике" (стр. 4) несправедливо. Методы оптимизации проектных решений и проектов хорошо известны и плодотворно используются на протяжении уже многих десятков лет (см. работы В.В. Титова, А.С. Плещинского, И.С. Межова и многие другие).
5) Можно ли гарантировать "...получение объективно оптимального, т.е. наилучшего варианта решения задачи оптимизации из всех возможных в заданных условиях" (см. стр. 5), если модель строится экономистом-аналитиком (субъектом)?

6) Что имелось в виду, когда автор утверждает, что "...Для определения состава и структуры такой системы показателей с точки зрения системного анализа необходимо инвестиционную деятельность представить как систему в форме модели «черного ящика»" (стр. 7)? Инвестиционная деятельность в виде "черного ящика"? Экономисты при анализе ИП заинтересованы как можно лучше знать свойства инвестиционного процесса (его финансовые потоки, ставки заимствования и внешнего использования, стоимость ресурсов и т.д.), т.е. работать с "белым ящиком" (ну, в крайнем случае - с "серым ящиком")!

7) Рис. 4 очень упрощенно иллюстрирует работу с финансовыми потоками проектов. На самом деле все обстоит гораздо сложнее (потоки являются динамическими, с запаздыванием выходных потоков по отношению к входным, зависят от внешних факторов и т.д.).

8) Утверждение "...Тогда эффективность этого процесса можно оценить в форме стоимостного показателя, определяющего алгебраическую сумму оттоков и притоков" (стр. 8) ошибочно (см. пояснения в пп. 7).

Не будем больше злоупотреблять вниманием читателя к ошибкам и неточностям рассматриваемой работы [21]. При желании, он может обратиться к оригиналу. Отметим только, что в целом хорошая идея по использованию системного подхода к задачам анализа ИП обернулась методиками, которыми практически в таком виде пользоваться нельзя. Что касается вопросов оптимизации ИП, то эта задача не такая простая, как утверждается в [21]. В частности, показатели эффективности проектов могут зависеть друг от друга, элементы выходного потока зависят от элементов входного потока в динамике и с лагом, многокритериальную задачу оптимизации нельзя решать методом нормализации критериев [27] и т.д. Некоторые из особенностей таких оптимизационных задач были рассмотрены выше.

Перечислим еще некоторые интересные с теоретической и практической точек зрения задачи анализа ИП: задача оптимизации структуры капитала ИП [22], [23], задача оценивания эффективности интегрированных проектов [24], задача выбора критериев эффективности ИП [25], [26],

\section{Выводы}


1. Рассмотрены проблемы предлагаемых методов анализа и синтеза ИП с учетом инфляционных процессов. Предложены схемы оценивания эффективности проектов с учетом инфляции для классических показателей NPV, IRR, DPP.

2. Выявлены недостатки некоторых схем оценивания срока окупаемости проектов.
3. Исследованы проблемы некоторых оптимизационных моделей для синтеза ИП.

4. Предложены подходы к оцениванию эффективности интегрированных проектов.

5. Выявлены проблемы методик использования системного подхода к анализу проектов.

\section{References:}

1. Vilenskiy PL, Livshits VN, Smolyak SA (2001) Otsenka effektivnosti investitsionnyih proektov: teoriya i praktika: uchebno-praktich. posobie. Moscow. Delo, pp. 451.

2. Lipsits IV, Kossov VV (2003) Ekonomicheskiy analiz realnyih investitsiy. Moscow. Ekonomist. pp. 341.

3. Rimer MI, Kasatov AD, Matienko NN (2008) Ekonomicheskaya otsenka investitsiy. SPb. Piter, pp. 480.

4. Kalmyikova TS (2009) Investitsionnyiy analiz: ucheb. posobie. Moscow. INFRA-M, pp. 204.

5. Kovalev VV (2005) Kurs finansovyih vyichisleniy: ucheb. posobie. Moscow. Finansyi i statistika, pp. 354.

6. Tsarev VV (2004) Otsenka ekonomicheskoy effektivnosti investitsiy. SPb. Piter. pp. 147.

7. Kryilov EI, Medvedeva SN (2003) Otsenka effektivnosti investitsiy $\mathrm{v}$ usloviyah inflyatsii. SPb. SPbGUAP. pp. 22.

8. Yakovleva NA (2000) Analiz effektivnosti investitsionnyih proektov (s uchetom faktora vremeni, riska i inflyatsii): Ucheb.-metod. posob. Mn. BGU. pp. 63.

9. Dosuzheva EE, Kirillov YV (2015) K voprosu ob uchete inflyatsii pri otsenke effektivnosti investitsionnyih proektov. International Scientific Journal Theoretical \& Applied Science, No. 4 (24), pp. 236-241. Soi: http://so-i.org/1.1/TAS*04(24)43 Doi: http://dx.doi.org/10.15863/TAS.2015.04.24.43

10. Naumov AA (2013) O tochnosti otsenok sroka okupaemosti investitsionnyih proektov. Theoretical\&Applied Science, Materials of the ISPC «Results \&Perspectives», 30.09.2013, Florence, Italy, No. 9 (5), pp. 95-99. doi: http://dx.doi.org/10.15863/TAS.2013.09.5.10

11. Naumov AA (2013) K voprosu o tochnosti otsenki diskontirovannogo sroka okupaemosti investitsionnogo proekta. Finansovaya analitika: problemyi i resheniya, No. 44 (182), pp. 25-28.
12. Naumov AA (2013) K analiticheskim resheniyam nekotoryih ekonomikomatematicheskih zadach. Theoretical\&Applied Science, Materials of the ISPC «Results \&Perspectives», 30.09.2013, Florence, Italy, No. 9 (5), pp. 99-104. doi: http://dx.doi.org/10.15863/TAS.2013.09.5.11

13. Naumov AA (2013) K voprosu ob uproschenii dvuh zadach optimizatsii investitsiy. Finansovaya analitika: problemyi i resheniya, No. 46 (184), pp. 26-30.

14. Naumov AA (2013) K modelyam sovmestnogo upravleniya proektami. Theoretical\&Applied Science, Materials of the ISPC «Theory and Practice», 30.08.2013, Munich, Germany, No. 8 (4), pp. 90-93. doi: http://dx.doi.org/10.15863/TAS.2013.08.4.11

15. Naumov AA, Kryukov SV (2013) K problemam odnoy modeli upravleniya sovmestnyimi investitsionnyimi proektami. Finansovaya analitika: problemyi i resheniya, No. 40 (178), pp. 53-56.

16. Naumov AA (2013) Ispolzovanie metoda detalizatsii finansovyih potokov $\mathrm{v}$ zadachah otsenivaniya effektivnosti proektov. Finansovaya analitika: problemyi i resheniya, No. 48 (186), pp. 35-41.

17. Naumov A.A. (2012) K zadache otsenivaniya chastnyih effektov integrirovannyih biznesprotsessov. Finansovaya analitika: Problemyi i resheniya, No. 46 (136), pp. 41-48.

18. Naumov AA (2012) Teoreticheskie i prikladnyie voprosyi modelirovaniya biznesprotsessov. Modeli, algoritmyi, programmyi. LAP LAMBERT Academic Publishing, pp. 464.

19. Naumov AA (2013) Metodyi analiza i sinteza investitsionnyih proektov. Effektivnost, riski, upravlenie. LAP LAMBERT Academic Publishing.

20. Spisok trudov. Available: https://sites.google.com/site/anatolynaumov201 
1/home/spisok-trudov-list-of-papers (Accessed 17.05.2015).

21. Dosuzheva EE (2015) Metodologiya sistemnogo podhoda i sistemnogo analiza $\mathrm{k}$ protsessu prinyatiya resheniy pri investirovanii. Sovremennyie nauchnyie issledovaniya i innovatsii, No. 5. Available: http://web.snauka.ru/issues/2015/05/53062 (Accessed: 24.05.2015).

22. Naumov AA, Naumova AA (2014) O nekorrektnosti odnoy modeli optimizatsii strukturyi kapitala. ISJ Theoretical\&Applied Science, Materials of the ISPC «European Innovation», 30.09.2014, Martigues, France, No. 9 (17), pp. 170-173. doi: http://dx.doi.org/10.15863/TAS.2014.09.17.29

23. Naumov AA (2013) Optimizatsiya strukturyi zaimstvovaniy i vlozheniy dohodov investitsionnogo proekta. Theoretical \& Applied Science, Materials of the ISPC «Advances in techniques\&technologies», 30.10.2013, Milan, Italy, No. 10(6), pp. 133136. doi: http://dx.doi.org/10.15863/TAS.2013.10.6.20
24. Naumov AA (2014) Otsenivanie effektivnosti integrirovannyih proektov. Finansovaya analitika: problemyi i resheniya, No. 8 (194), pp. 36-43.

25. Naumov AA (2014) Analiz kriteriev effektivnosti investitsionnyih proektov. ISJ Theoretical\&Applied Science, Materials of the ISPC «Modern mathematics in science», 30.06.2014, Caracas, Venezuela, No. 6 (14), pp. 92-94. doi: http://dx.doi.org/10.15863/TAS.2014.06.14.19

26. Naumov AA, Bazhenov RI (2014) O problemah klassicheskih pokazateley effektivnosti investitsionnyih proektov. Sovremennyie nauchnyie issledovaniya i innovatsii, No. 11. Available:

http://web.snauka.ru/issues/2014/11/40825 (Accessed: 20.11.2014).

27. Naumov AA, Bazhenov RI (2014) O neustoychivosti metoda normalizatsii kriteriev. Sovremennyie nauchnyie issledovaniya i innovatsii, No. $11 . \quad$ Available: http://web.snauka.ru/issues/2014/11/40408 (Accessed: 10.11.2014). 